\title{
Compensating Temperature-Dependent Characteristics of a Subthreshold-MOSFET Analog Silicon Neuron
}

\author{
Ethan Green \\ Department of Electrical Engineering and Information Systems, The University of Tokyo, 4-6-1 Komaba, \\ Meguro-ku, Tokyo, 153-8505, Japan \\ Takashi Kohno \\ Institute of Industrial Science, The University of Tokyo, 4-6-1 Komaba, \\ Meguro-ku, Tokyo, 153-8505, Japan \\ E-mail: green@sat.t.u-tokyo.ac.jp,kohno@sat.t.u-tokyo.ac.jp \\ www.u-tokyo.ac.jp
}

\begin{abstract}
Analog silicon neurons are neuro-mimetic VLSI (very-large-scale-integrated) circuits that replicate the electrophysiological behavior of animal nerve tissue. This research focuses on the temperature sensitivity of a subthreshold-MOSFET analog silicon neuron. Subthreshold operation of CMOS transistors allows for low power consumption, but is also drastically sensitive to temperature changes. This critical issue must be addressed before these circuits can be implemented into massive networks to develop future neuromorphic technologies.
\end{abstract}

Keywords: neuromorphic engineering, analog VLSI, silicon neurons

\section{Introduction}

The field of neuromorphic engineering seeks to design circuits that mimic the electrophysiological behavior and network structure of neurons to create biologicallyinspired or neuromimetic technology that can perform calculations in ways fundamentally different from traditional digital computers. Motivations for research in this field include developing brain-like computers and bio-silico hybrid systems for medical devices.

Analog silicon neurons are electronic circuits that exploit the characteristics of transistors to mimic how nerve cells control membrane potential. These circuits operate in continuous time, can replicate a variety of spike shapes and spiking behavior, and require low power ${ }^{1,2,3,4}$. They are expected to be a promising tool to construct neuromorphic systems. However, many technical challenges must be addressed before these circuits can be implemented in large-scale networks. A possible method to solve a key issue, temperature sensitivity, is to actively tune the circuit parameters depending on temperature. We report our approach in which appropriate parameter sets for several temperatures are found and parameter sets for intermediary temperatures are obtained by interpolation.

\section{Circuit Description}

The circuit used in this research (Fig. 1) is an ultra-low power VLSI silicon neuron based on qualitative neuronal modeling ${ }^{1}$, which seeks to use judicious approximation and mathematical understanding ${ }^{5}$ to convert the model of membrane potential to equations with fewer variables that are more suitable for circuit implementation. In the model for this circuit, variable $v$ represents membrane potential and variable $n$ represents abstracted ionic activity. The value of each variable is calculated by subtracting the voltage over each capacitor from $V_{d d}(1.0 \mathrm{~V})$. These two variables control the output currents of transconductance circuit components $f_{x}(v), g_{x}(v)(x=v, n)$, and $r(n)$ that charge and discharge capacitors attached to the $v$-block and $n$-block within the circuit. This circuit was shown to support the dynamical behaviors of Class I and II neurons in the Hodgkin's classification ${ }^{6}$. The system equations of this model are as follows: 


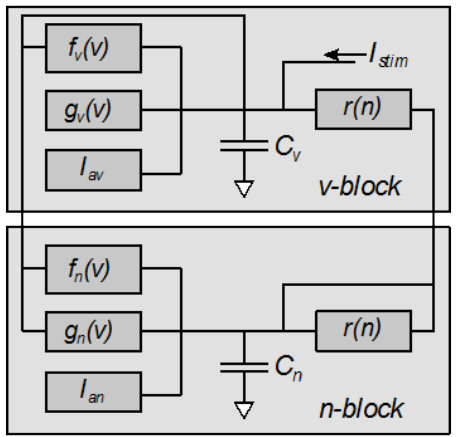

Fig. 1. Circuit block diagram of the silicon neuron circuit.

$C_{v} \frac{d v}{d t}=f_{v}(v)-g_{v}(v)+I_{a v}-r(n)+I_{s t i m}$

$C_{n} \frac{d v}{d t}=f_{n}(v)-g_{n}(v)+I_{a n}-r(n)$

$I_{\text {stim }}$ is an external stimulus current, and $I_{a v}$ and $I_{a n}$ are constant currents. The current-voltage characteristics of the $f_{x}(v), g_{x}(v) \quad(x=v, n)$, and $r(n)$ components are expressed by the following sigmoidal relationships:

$$
\begin{aligned}
& f_{x}(v)=M_{x} /\left(1+\exp \left(-\frac{\kappa}{U_{T}}\left(v-\delta_{x}\right)\right)\right) \\
& g_{x}(v)=I_{0} \sqrt{\exp \left(\frac{\kappa}{U_{T}} \theta_{x}\right) /\left(1+\exp \left(-\frac{\kappa}{U_{T}}\left(v-\theta_{x}\right)\right)\right)} \\
& r(n)=I_{0} \sqrt{\exp \left(\frac{\kappa}{U_{T}} \theta_{r}\right) /\left(1+\exp \left(-\frac{\kappa}{U_{T}}\left(v-\theta_{r}\right)\right)\right)}
\end{aligned}
$$

$I_{0}$ is the PMOS transistor off-current, $U_{T}$ is the thermal voltage ( $\sim 26 \mathrm{mV}$ at room temperature), and $\kappa$ is the capacitive coupling ratio. Parameters $M_{x}, \theta_{x}, \delta_{x}(x=v, n)$, and $\theta_{r}$ can be tuned by bias voltages applied to the circuit. All transistors are operated in the subthreshold regime. Figure 2 (a) shows a differential pair attached to a current mirror used for $f_{v}(v)$ and $f_{n}(v)$. Figure 2 (b) shows the circuit used for $g_{v}(v), g_{n}(v)$, and $r(n)$. This circuit is a cascoded transistor with source degeneration and a detached bulk voltage.

This silicon neuron circuit includes a nullclinedrawing function which assists in the diagramming of a phase plane to evaluate the circuit's dynamical structure. For example, a sigmoidally shaped $n$-nullcline and cubic-shaped $v$-nullcline that cross at 3 distinct points yields Class I behavior in the Hodgkin's

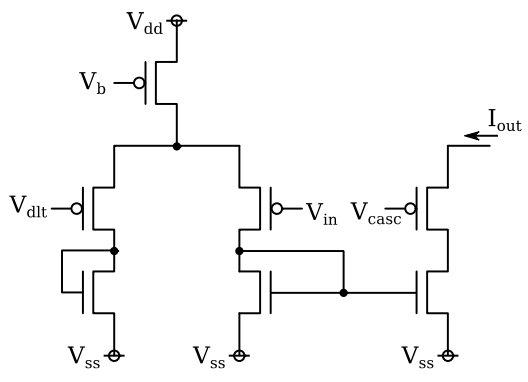

(a)

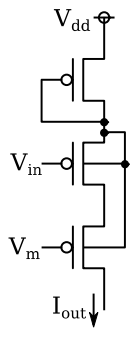

(b)
Fig. 2. Circuit components. (a) $f_{v}(v)$ and $f_{n}(v)$, (b) $g_{v}(v), g_{n}(v)$, and $r(n)$.

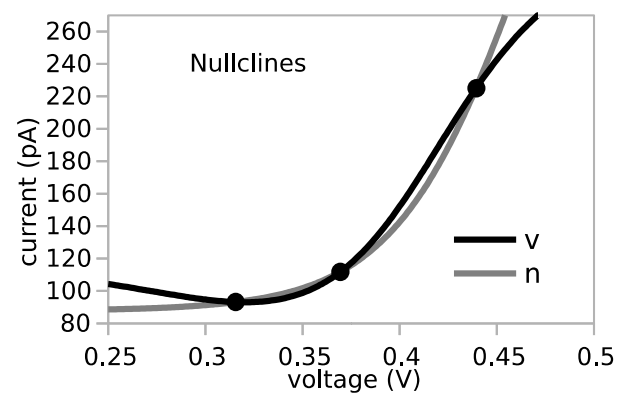

Fig. 3. Nullclines in Class I mode.

classification (Fig. 3). The points are characterized mathematically through analysis with nonlinear dynamics ${ }^{5}$.

The temperature sensitivity of this circuit originates in the intrinsic temperature sensitivity of MOSFETs in the subthreshold regime. Figure 4 illustrates periodic spiking behavior in the Class I mode in response to a 4 pA sustained stimulus over a narrow range of temperatures from 25 to $26.5^{\circ} \mathrm{C}$. The stimulus begins at 0.2 seconds. As can be seen from the figure, the spiking frequency varies by temperature, and ceases at $26.5^{\circ} \mathrm{C}$.

\section{Parameter Compensation by Interpolation}

In this work, we suppose a temperature sensitivity management algorithm in which the parameter voltages applied to the circuit are adjusted depending on the temperature. Such an algorithm will be used by simple on-chip components to control circuit parameters, thus complementing the low-power characteristics of the circuit. We examined an approach to finding appropriate parameter sets for each temperature which maintain the 


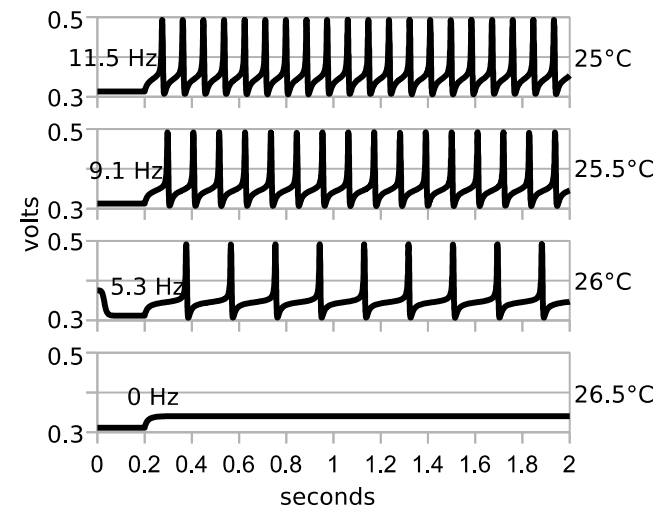

Fig. 4. Periodic spiking at different temperatures.

important characteristics of a neuron: spike frequency in response to sustained stimuli and the threshold pulse stimulus for spike generation.

First, circuit behavior was analyzed at $27^{\circ} \mathrm{C}$ and these results were considered the benchmark for operation at other temperatures. The data recorded included frequency response to $5 \mathrm{pA}$ and $10 \mathrm{pA}$ sustained stimuli, and the threshold current $I_{t h}$ of a pulse stimulus $(500 \mu$ s pulse width) required to generate an action potential. The $v$ and $n$-nullclines were also plotted. Next, the circuit was simulated at 17, 22, 32, and $37^{\circ} \mathrm{C}$. Selected circuit parameters were tuned to generate behavior that most closely matched the $27^{\circ} \mathrm{C}$ benchmark. The nullcline-drawing function was used extensively, with the conjecture that similarly shaped nullclines lead to similar circuit behavior. Finally, the tuned parameter sets were input into a script which interpolates functions from these data points and returns circuit parameter sets for any intermediary temperature. This procedure was performed with the Class I setting simulated by the Spectre circuit simulator.

\section{Simulation Results}

\subsection{Simple approach}

We selected 5 significant circuit parameters because exploring high-dimensional parameter space is inefficient. The tail current of the $f_{n}(v)$ component, the bias voltages of the $g_{v}(v)$ and $r(n)$ components, and the constant current sources $I_{a v}$ and $I_{a n}$ were tuned with all other circuit parameters held constant. The $g_{n}(v)$ component was turned off. Table 1 shows the best parameter sets which most closely replicated the benchmark behavior at each temperature. The bottom 3 rows of the table show the circuit behavior at each temperature. The script was then used to generate parameter sets for all intermediary temperatures in $0.5^{\circ} \mathrm{C}$ steps from 17 to $37^{\circ} \mathrm{C}$. Parameters were rounded to the nearest $0.5 \mathrm{mV}$. These 42 parameter sets were then simulated with Spectre in their corresponding temperatures. The "simple" column in Table 2 summarizes the results. "Average \% change" refers to the average percent difference from the benchmark behavior. Firing frequencies were relatively close to the benchmark behavior, with the exception of the $5 \mathrm{pA}$ stimulus at $29^{\circ} \mathrm{C}$ which failed to fire. The threshold current showed a downward trend with higher values above $200 \mathrm{pA}$ at lower temperatures and lower values around $140 \mathrm{pA}$ at higher temperatures.

Table 1. Parameter sets with corresponding circuit behavior using the simple approach

\begin{tabular}{|l|r|r|r|r|r|}
\hline Temperature $\left({ }^{\circ} \mathbf{C}\right)$ & $\mathbf{1 7}$ & $\mathbf{2 2}$ & $\mathbf{2 7}$ & $\mathbf{3 2}$ & $\mathbf{3 7}$ \\
\hline gv_Vm $(\mathrm{mV})$ & 388 & 417 & 432 & 443 & 449.5 \\
\hline fn_Vb $(\mathrm{mV})$ & 257 & 248 & 237 & 231 & 219 \\
\hline Iav_Vin $(\mathrm{mV})$ & 434 & 450 & 461 & 468.5 & 473.5 \\
\hline Ian_Vin $(\mathrm{mV})$ & 420 & 449.5 & 464.5 & 475 & 481.5 \\
\hline rn_Vm $(\mathrm{mV})$ & 480.5 & 462.5 & 445 & 430 & 415 \\
\hline$I_{\text {th }}(\mathrm{pA})$ & 201 & 185 & 178.5 & 164 & 147 \\
\hline $5 \mathrm{pA}$ response $(\mathrm{Hz})$ & 16.3 & 15.8 & 15.1 & 15.7 & 18.5 \\
\hline $10 \mathrm{pA}$ response $(\mathrm{Hz})$ & 39 & 38.8 & 36.2 & 38.7 & 39.4 \\
\hline
\end{tabular}

Table 2. Analysis of interpolation results

\begin{tabular}{|l|l|l|}
\hline & simple & full \\
\hline 5 pA stim average freq. $(\mathrm{Hz})$ & 15.8 & 11.9 \\
\hline 5 pA stim average $\%$ change & 5.2 & -9.3 \\
\hline 5 pA stim frequency range $(\mathrm{Hz})$ & $3.7-27.1$ & $2.4-26.1$ \\
\hline 10 pA stim average freq. $(\mathrm{Hz})$ & 38.7 & 35.5 \\
\hline 10 pA stim average \% change & 6.8 & -0.6 \\
\hline 10 pA stim freq. range $(\mathrm{Hz})$ & $34.6-43.2$ & $26.8-40.4$ \\
\hline Average threshold current $I_{\text {th }}(\mathrm{pA})$ & 172 & 185.4 \\
\hline Average $I_{\text {th }} \%$ change & -3.6 & -0.1 \\
\hline$I_{\text {th }}$ range $(\mathrm{pA})$ & $115.5-216$ & $131-303.5$ \\
\hline
\end{tabular}


Table 3. Parameter sets with corresponding circuit behavior using the full-parameter approach

\begin{tabular}{|l|r|r|r|r|r|}
\hline Temperature $\left({ }^{\circ} \mathbf{C}\right)$ & $\mathbf{1 7}$ & $\mathbf{2 2}$ & $\mathbf{2 7}$ & $\mathbf{3 2}$ & $\mathbf{3 7}$ \\
\hline fv_Vb $(\mathrm{mV})$ & 256.5 & 248.5 & 238 & 230 & 219 \\
\hline fv_Vdlt $(\mathrm{mV})$ & 567 & 571 & 580 & 588 & 596 \\
\hline gv_Vm $(\mathrm{mV})$ & 464 & 451 & 432 & 420 & 398 \\
\hline fn_Vb $(\mathrm{mV})$ & 255 & 246 & 237 & 228.5 & 219 \\
\hline fn_Vdlt $(\mathrm{mV})$ & 517 & 520 & 520 & 521 & 524 \\
\hline Iav_Vin $(\mathrm{mV})$ & 437 & 452 & 461 & 469 & 472 \\
\hline gn_Vm $(\mathrm{mV})$ & 220 & 178.5 & 156 & 132.5 & 113 \\
\hline Ian_Vin $(\mathrm{mV})$ & 395 & 445.5 & 461 & 473 & 479 \\
\hline rn_Vm $(\mathrm{mV})$ & 480.5 & 463.5 & 445 & 427 & 413 \\
\hline$I_{\text {th }}(\mathrm{pA})$ & 250 & 191 & 186 & 169 & 145 \\
\hline 5 pA response $(\mathrm{Hz})$ & 13.3 & 17.1 & 13.2 & 13.2 & 13.2 \\
\hline $10 \mathrm{pA}$ response $(\mathrm{Hz})$ & 32.7 & 36.5 & 35.7 & 36.0 & 38.6 \\
\hline
\end{tabular}

\subsection{Full-parameter approach}

A second approach augments the strategy used above by incorporating all the influential circuit parameters including the tail current and offset voltage of $f_{v}(v)$, the offset voltage of $f_{n}(v)$, and the bias voltage of $g_{n}(v)$. First, each of the circuit components were simulated individually and circuit parameters were sought that maintained $I-V$ characteristics over a range of temperatures. These parameters were then used as a starting point for tuning the entire circuit at different temperatures. Focus was placed on matching the nullclines as closely as possible. Through analysis of the individual components, it was discovered that the $g_{v}(v)$, and $g_{n}(v)$ components experience a temperature induced offset, which can be compensated for by tuning the offset voltages of the $f_{v}(v)$ and $f_{n}(v)$ components.

The parameters for the pillar temperatures are listed in Table 3. Those for the intermediary temperatures in the range $17-37^{\circ} \mathrm{C}$ in $0.5^{\circ} \mathrm{C}$ steps were determined in the same way as in the previous approach. The "full" column in Table 2 shows the results of the Spectre simulations. $I_{t h}$ showed a downward trend and the firing frequencies were relatively close to the benchmark, but the $5 \mathrm{pA}$ sustained stimulus failed to induce periodic spiking at $17.5-21^{\circ}, 29.5^{\circ}, 34.5^{\circ}, 35.5^{\circ}$, and $36.5^{\circ} \mathrm{C}$. While the temperature compensation with this approach was less effective, nullclines that more accurately matched the benchmark were obtained, suggesting that future modifications may yield better results.

\section{Discussion}

The above results can be improved by setting the interpolation script to return parameters rounded to the nearest $0.1 \mathrm{mV}$, but this accuracy would be difficult to replicate with real-world circuits because of thermal noise and other restrictions including the limited precision of bias voltage generator circuits.

The relative success of the simple approach suggests that limiting the available parameters leads to a smoother progression of values over a range of temperatures, allowing more success in intermediary steps. The full-parameter approach in contrast offered too much fine tuning capability. At a given temperature, some parameters were tuned more aggressively than others, depending on which yielded the most desirable behavior. This is a potential cause of the problems in the intermediary steps. Using a meta-heuristic algorithm to obtain the pillar parameter sets may solve this problem.

\section{Acknowledgements}

This study was supported by JST PRESTO and VLSI Design and Education Centre (VDEC) at the University of Tokyo with collaboration from Cadence Corporation.

\section{References}

1. T. Kohno and K. Aihara, "A Qualitative-Modeling-Based Low-Power Silicon Nerve Membrane," Electronics, Circuits, and Systems (ICECS), IEEE, pp. 199-202, December, 2014.

2. S. Liu, et al., Analog VLSI: Circuits and Principles, MIT Press, 2002.

3. E. Chicca, F. Stefanini, C. Bartolozzi, and G. Indiveri, "Neuromorphic electronic circuits for building autonomous cognitive systems," Proceedings of the IEEE, 102, 9, pp. 1367-1388, 2014.

4. S. Brink, S. Nease, and P. Hasler, "Computing with networks of spiking neurons on a biophysically motivated floating-gate based neuromorphic integrated circuit," Neural Networks, 45, pp. 39-49, 2013.

5. J. Rinzel and B. Ermentrout, "Analysis of Neural Excitability and Oscillations," Methods in Neuronal Modeling, Massachusetts Institute of Technology, pp. 251-291, 1998.

6. A. Hodgkin, "The local electric changes associated with repetitive action in a non-medullated axon," The Journal of Physiology, 107, 2, pp. 165-181, March, 1948. 